\title{
Financial Data Analysis by means of Coupled Continuous-Time Random Walk in Rachev-Rüschendorf Model
}

\author{
A. Jurlewicz ${ }^{a, *}$, A. WyŁomańska ${ }^{a, \dagger}$ And P. ŻEBrowski ${ }^{b}$ \\ ${ }^{a}$ Hugo Steinhaus Center \\ Institute of Mathematics and Computer Science \\ Wrocław University of Technology \\ Wyb. Wyspiańskiego 27, 50-370 Wrocław, Poland \\ ${ }^{b}$ Mathematical Institute, University of Wrocław \\ pl. Grunwaldzki 2/4, 50-348 Wrocław, Poland
}

We adapt the continuous-time random walk formalism to describe asset price evolution. We expand the idea proposed by Rachev and Rüschendorf who analyzed the binomial pricing model in the discrete time with randomization of the number of price changes. As a result, in the framework of the proposed model we obtain a mixture of the Gaussian and a generalized arcsine laws as the limiting distribution of log-returns. Moreover, we derive an European-call-option price that is an extension of the Black-Scholes formula. We apply the obtained theoretical results to model actual financial data and try to show that the continuous-time random walk offers alternative tools to deal with several complex issues of financial markets.

PACS numbers: 89.65.Gh, 05.40.Fb

\section{Introduction}

The observed distributions of returns for financial assets are central elements in pricing of securities like options [1]. Option pricing theory has a long and illustrious history. It underwent a revolutionary change in 1973 when Black and Scholes presented the first completely satisfactory equilibrium option pricing model [2]. Afterwards, in 1979 Cox et al. proposed a new idea of looking at the binomial pricing model as a discrete-time approximation to continuous-time diffusion [3]. In the classical Cox-Ross-Rubinstein (CRR) binomial model of the market one assumes $[3,4]$ that in time interval $[0, T]$ (for a fixed time $T$ ) the value of the stock price changes $n$ times at instants of time $k T / n, k=1, \ldots n$, according to the

\footnotetext{
${ }^{*}$ corresponding author; e-mail: Agnieszka.Jurlewicz@pwr.wroc.pl

${ }^{\dagger}$ This research was supported by the Deutsche Forschungsgemainschaft through the SFB "Economic Risk".
} 
rule $S_{k}=u_{n}^{\epsilon_{n, k}} d_{n}^{1-\epsilon_{n, k}} S_{k-1}$, where the initial value $S_{0}$ is a positive constant, and $S_{n}=S(T)$ is the stock price at the considered time $T$. The introduced sequence $\left(\epsilon_{n, k}\right)_{k \geq 1}$ consists of independent and identically distributed (i.i.d.) random variables taking values 1 and 0 with probabilities $p_{n}$ and $1-p_{n}$, respectively, for some $0<p_{n}<1$. Parameters $u_{n}>1,0<d_{n}<1$ together with $\left(\epsilon_{n, k}\right)_{k \geq 1}$ reflect stock-price ups and downs. The log-returns at time $T$ result from $n$ i.i.d. jumps represented by $X_{n, k}, k \geq 1$ :

$$
\log \left(S(T) / S_{0}\right)=\sum_{k=1}^{n} X_{n, k}
$$

where $\left(X_{n, k}\right)_{k \geq 1}$ is a sequence of i.i.d. random variables taking values $\log u_{n}$ and $\log d_{n}$ with probabilities $p_{n}$ and $1-p_{n}$, respectively.

Usually, one assumes $\log u_{n}=\tilde{\sigma} \sqrt{T / n}=-\log d_{n}$ and $p_{n}=\frac{1}{2}+\frac{\alpha}{2 \tilde{\sigma}} \sqrt{\frac{T}{n}}$ with some $\alpha \in \mathbb{R}$ and volatility $\tilde{\sigma}>0$ such that $\tilde{\sigma}^{2}>2 r$ for the short-term interest rate $r>0$. Such a choice of parameters $u_{n}, d_{n}, p_{n}$ with increasing number $n$ of price changes yields [3] asymptotically Gaussian distribution $\mathcal{N}\left(\alpha T, \tilde{\sigma}^{2} T\right)$ of log-returns (1), and, as a consequence, the famous Black-Scholes formula for the rational price $C_{0}$ of a standard European call option with the maturity time $T$ and the strike price $K[4-10]$.

The empirical distributions of many real-life financial data essentially deviate from the Gaussian law provided by CRR model, see e.g. [11, 12]. In order to find more adequate limiting models, in 1994 Rachev and Rüschendorf extended the CRR conception by introducing two additional randomizations in the binomial pricing model $[13,14]$. One of the Rachev-Rüschendorf (RR) asset-pricing models assumes that the number of price changes in the time interval $[0, T]$ is not $n$ but a random variable $N_{n}$ that is independent of sequence $\left(X_{n, k}\right)_{k \geq 1}$, representing the price jumps. In this paper, motivated by the continuous-time random walk (CTRW) methodology, we expand the RR model by introducing a clustering scheme for defining $N_{n}$.

CTRW processes (i.e. random walks with random time intervals between subsequent jumps) have been extensively studied [15-25] as a useful mathematical tool for description and analysis of phenomena connected with anomalous diffusion, e.g. relaxation processes, fully developed turbulence, transport in disordered or fractal media. Recently, they have been applied also in finance to model the movement of log-prices and the risk process $[12,26,27]$.

Within the CTRW methodology, the analysis of the diffusion-front properties based directly on the definition of the CTRW as a cumulative stochastic process, and performed by means of limit theorems of probability theory, leads to the total-distance limiting distributions related not only to the Gaussian and Lévy-stable laws but also to the generalized arcsine distribution (i.e. beta distribution with parameters $p=1-q)[20-22,28,29]$. The latter results from the assumed dependence between jumps and waiting times provided by a random 
clustering procedure, introduced to model relaxation phenomena [21, 22, 29].

In this paper, in Sect. 2 we propose similar clustering scheme for a simple CTRW-like log-returns (1). Such a procedure, that roughly speaking means assembling/clustering jumps in some "packets" of random sizes, leads to a special randomization of the number of stock-price changes in the RR model. In Sect. 3 we present a limiting distribution of the resulting log-returns and the corresponding pricing formula for the European call option. Then, in Sect. 4 we apply the obtained results in description of real financial data in comparison with the classical fitting by the Gaussian law.

\section{Clustering procedure}

We take $\left(M_{j}\right)_{j \geq 1}$, a sequence of i.i.d. random indices (i.e. positive integer-valued random variables), independent of $\left(X_{k, n}\right)_{k, n \geq 1}$. Then, by means of $\left(M_{j}\right)$, we choose instants of time $\tau_{i}=\mu_{i} T / n$, where $\mu_{0}=0, \mu_{i}=\sum_{j=1}^{i} M_{j}$ for $i \geq 1$. The succesive stock-price ups and downs performed (in CRR model) after time $\tau_{i-1}$ and before or at time $\tau_{i}$ are represented by cumulative jump $\tilde{X}_{n, i}=\sum_{k=\mu_{i-1}+1}^{\mu_{i}} X_{n, k}$ performed at time $\tau_{i}$. The random variable $M_{i}$ corresponds hence to the size of the $i$-th packet of the price changes, and $\tilde{X}_{n, i}$ refers to the cumulative price change for this packet.

The proposed clustering procedure is connected with a hedging strategy represented by European call option [10]. Namely, at the beginning of the considered time period $\left(\tau_{i-1}, \tau_{i}\right]$ we predict its length (by means of $\left.M_{i}\right)$, but we get the cumulative value of the asset prices not before the end of this period, after the corresponding CRR jumps occurred. This situation can be adequate to the case when we do not observe the asset prices at any available time point but monitor the value at randomly chosen instants of time. Therefore, to predict the option price on time $T$ we dispose, in fact, only information until the latest of the time instants $\tau_{i}$ before or at $T$. For such a strategy, the resulting log-returns at time $T$ are given by

$$
\log \left(S(T) / S_{0}\right)=\sum_{k=1}^{K_{n}} \tilde{X}_{n, k}=\sum_{k=1}^{N_{n}} X_{n, k},
$$

where $K_{n}=\max \left\{k \in \mathbb{N}: \mu_{k} \leq n\right\}$, and

$$
N_{n}=\mu_{K_{n}}=\sum_{j=1}^{K_{n}} M_{j} .
$$

Formula (2) is consistent with general assumptions of RR model with randomized number of price changes. In the proposed model, however, $N_{n}$ is of the special form (3).

\section{Limiting distribution of log-returns. Option pricing formula}

Applying general results for RR model [7] and well-known facts of the renewal theory $[30,31]$ we obtain the limiting distribution of log-returns and the resulting 
formula for the rational price of a standard European call option in the framework of the proposed construction of number $N_{n}$ of price jumps. If the random packet sizes are of finite mean value (i.e. $\left\langle M_{j}\right\rangle<\infty$ ), the proposed clustering scheme does not go beyond the results derived in the framework of CRR model; in particular, beyond the asymptotically Gaussian distribution of log-returns and the BlackScholes pricing formula. On the other hand, an interesting extension of the classical results can be obtained if we take into account heavy-tailed random packet sizes i.e. the case when for some $0<\gamma<1$ we have $\operatorname{Pr}\left(M_{1}>m\right) \sim m^{-\gamma}$ for large $m$. Then for $n \rightarrow \infty$ the limiting distribution of log-returns (2) becomes a mixture of the Gaussian and a generalized arcsine distributions. The probability density function of this mixed law reads as

$$
f_{T}(x ; \alpha, \gamma)=\frac{\sin (\pi \gamma)}{\sqrt{2 \pi^{3} \tilde{\sigma}^{2} T}} \int_{0}^{1} \exp \left(-\frac{(x-\alpha T y)^{2}}{2 y \tilde{\sigma}^{2} T}\right) \frac{y^{\gamma-3 / 2} \mathrm{~d} y}{(1-y)^{\gamma}} .
$$

Moreover, the resulting limiting European call option price (with the maturity time $T$ and the strike price $K$ ) is given by the formula

$$
\begin{array}{r}
C_{0}(\alpha, \gamma)=S_{0} \frac{\sin (\pi \gamma)}{\pi} \int_{0}^{1} \Phi\left(\frac{\log \left(S_{0} / K\right)+\left(r+\frac{1}{2} \tilde{\sigma}^{2}\right) T y}{\tilde{\sigma} \sqrt{T y}}\right) \frac{y^{\gamma-1} \mathrm{~d} y}{(1-y)^{\gamma}} \\
-K \frac{\sin (\pi \gamma)}{\pi} \int_{0}^{1} \mathrm{e}^{-r T y} \Phi\left(\frac{\log \left(S_{0} / K\right)+\left(r-\frac{1}{2} \tilde{\sigma}^{2}\right) T y}{\tilde{\sigma} \sqrt{T y}}\right) \frac{y^{\gamma-1} \mathrm{~d} y}{(1-y)^{\gamma}}
\end{array}
$$

that is an essential extension of the classical Black-Scholes formula.

\section{Real-data analysis}

We apply now the presented RR model with $N_{n}$ of form (3) in description of real financial data (data of daily log-returns of Standard \& Poor's 500 index from years 1950-2005, daily log-retturns on NASDAQ value weighted index from years 1990-2005, and monthly log-returns on prices of 7-year bonds from years 1946-2006). The empirical distributions of log-returns fitted by the obtained limiting distribution (4) in comparison with the Gaussian distribution are presented in Figs. 1-3. The evaluated best-fit parameters and values $K$ of the Kolmogorov statistics are collected in Table. As we see, the mixed limiting law (4) gives a satisfactory approximation of modelled distributions of various financial instruments. For each considered data the value of the Kolmogorov statistics is smaller for the mixed limiting law (4) than for the Gaussian law. 


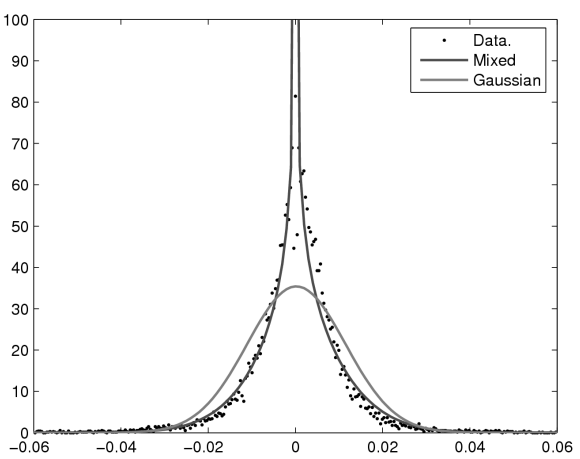

Fig. 1. Standard \& Poor's 500 data fitted by the mixed and Gaussian laws with parameters from Table $(T=1$ day).

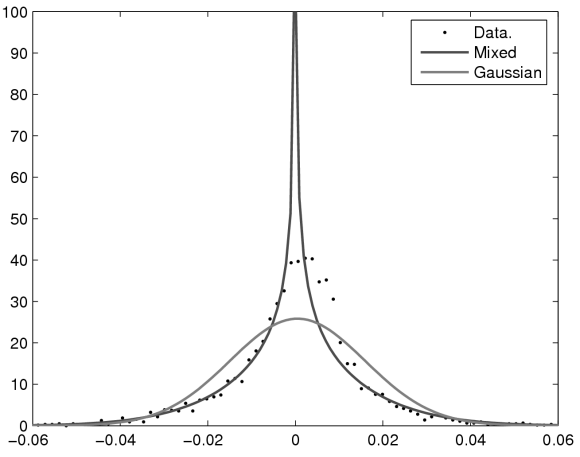

Fig. 2. NASDAQ data fitted by the mixed and Gaussian laws with parameters from Table ( $T=1$ day).

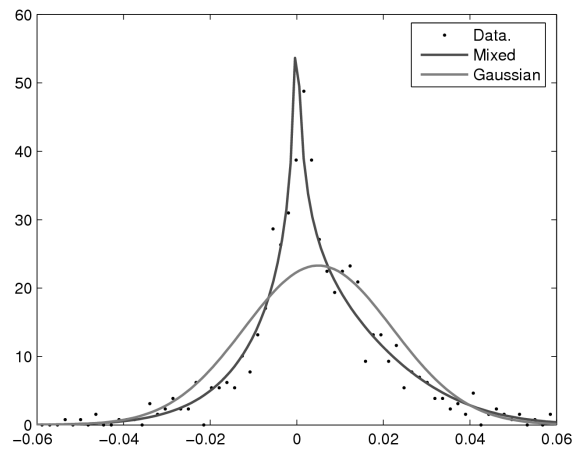

Fig. 3. 7-year bonds data fitted by the mixed and Gaussian laws with parameters from Table $(T=1$ month). 
TABLE

The best-fit parameters and values $\boldsymbol{K}$ of the Kolmogorov statistics for the mixed law (4) and the Gaussian law fitting the empirical distributions of daily log-returns on Standard \& Poor's 500 index, NASDAQ index, and 7-year bonds.

\begin{tabular}{c|c|c|c|c}
\hline \hline \multicolumn{2}{c|}{ Data } & S\&P 500 & NASDAQ & 7-year bonds \\
\hline \multirow{3}{*}{ mixed } & $\gamma$ & 0.47 & 0.40 & 0.5 \\
law & $\alpha$ & 0.365 & 0 & 0.12 \\
& $\tilde{\sigma}$ & 0.2675 & 0.4394 & 0.0762 \\
& $\boldsymbol{K}$ & $\mathbf{0 . 0 2 2 5}$ & $\mathbf{0 . 0 6 1}$ & $\mathbf{0 . 0 1 8}$ \\
\hline Gaussian & $\alpha$ & 0.0828 & 0.1822 & 0.06 \\
law & $\tilde{\sigma}$ & 0.2158 & 0.2942 & 0.0592 \\
& $\boldsymbol{K}$ & $\mathbf{0 . 0 9 2 9}$ & $\mathbf{0 . 0 8 2}$ & $\mathbf{0 . 0 7 2 7}$
\end{tabular}

\section{References}

[1] R. Merton, Continuous Time Finance, Blackwell, Oxford, U.K. 1993.

[2] F. Black, M. Scholes, J. Political Economy 81, 637 (1973).

[3] J.C. Cox, S.A. Ross, M. Rubinstein, J. Finance Econ. 7, 229 (1979).

[4] A.N. Shiryaev, Essentials of Stochastic Finance: Facts, Models, Theory, World Sci., Singapore 1999.

[5] A. Rejman, A. Weron, R. Weron, Stochastic Models 13, 867 (1997).

[6] J. Laurent, D. Leisen, in: Quantitative Analysis in Financial Markets, Ed. M. Avellaneda, World Sci., New York 2000, p. 216.

[7] S. Rachev, S. Mittnik, Stable Paretian Models in Finance, Wiley, New York 2000.

[8] D. Bertsimas, I. Popescu, Operations Res. 50, 358 (2002).

[9] R. Weron, Probabil. Math. Statist. 22, 417 (2002).

[10] J.C. Hull, Options, Futures and Other Derivatives, 6th ed., Prentice Hall, London 2005.

[11] M. Bellalah, M. Lavielle, Multinational Finance J. 6, 99 (2002).

[12] M.M. Meerschaert, E. Scalas, Physica A 370, 114 (2006).

[13] S. Rachev, L. Rüschendorf, Theory Probab. Appl. 39, 120 (1994).

[14] L. Rüschendorf, Tr. Mian. 237, 143 (2002).

[15] R. Metzler, J. Klafter, Phys. Rep. 339, 1 (2000).

[16] I.M. Sokolov, Phys. Rev. E 63, 011104 (2000); Phys. Rev. E 66, 041101 (2002).

[17] E. Gudowska-Nowak, K. Weron, Phys. Rev. E 65, 011103 (2001).

[18] A.A. Stanislavsky, Theor. Math. Phys. 138, 418 (2004).

[19] E. Gudowska-Nowak, K. Bochenek, A. Jurlewicz, K. Weron, Phys. Rev. E 72, 061101 (2005). 
[20] A. Piryatinska, A.I. Saichev, W.A. Woyczynski, Physica A 349, 375 (2005).

[21] K. Weron, A. Jurlewicz, Def. Diff. Forum 237-240, 1093 (2005).

[22] K. Weron, A. Jurlewicz, M. Magdziarz, Acta Phys. Pol. B 36, 1855 (2005).

[23] M. Magdziarz, K. Weron, Acta Phys. Pol. B 37, 1617 (2006).

[24] M. Magdziarz, A. Weron, K. Weron, Phys. Rev. E 75, 016708 (2007).

[25] M. Magdziarz, A. Weron, Phys. Rev. E 75, 056702 (2007); 76, 066708 (2007).

[26] E. Scalas, Physica A 362, 225 (2006).

[27] M. Magdziarz, P. Miśta, A. Weron, Acta Phys. Pol. B 38, 1647 (2007).

[28] M.M. Meerschaert, H.-P. Scheffler, J. Appl. Probab. 41, 623 (2004).

[29] A. Jurlewicz, Diss. Math. 431, 1 (2005).

[30] W. Feller, An Introduction to Probability Theory and Its Applications, Vol. 2, Wiley, New York 1966.

[31] A. Gut, Stopped Random Walks. Limit Theorems and Applications, Springer, New York 1988. 